\title{
Resonant two-photon single ionization of two atoms
}

\author{
C. Müller and A. B. Voitkiv \\ Max-Planck-Institut für Kernphysik, Saupfercheckweg 1, 69117 Heidelberg, Germany
}

(Dated: November 9, 2018)

\begin{abstract}
Resonant two-photon ionization in a system consisting of two spatially well-separated atoms is studied. Due to two-center electron-electron correlations, the ionization may also proceed through photo-excitation of both atoms with subsequent interatomic Coulombic decay. We show that this channel may dominate the photoionization process and qualitatively change its dependence on the field intensity and the spectra of emitted electrons.

PACS numbers: $32.80 . \mathrm{Rm}, 32.80 . \mathrm{Zb}, 42.50 . \mathrm{Hz}$
\end{abstract}

The development of tunable laser sources in the 1970s stimulated in-depth studies of the behavior of atoms and molecules exposed to intense resonant electromagnetic fields [1, 2]. While the emission of fluorescence light can provide detailed atomic structure information, resonance-enhanced multiphoton ionization has become a valuable tool for probing dynamical aspects of atoms, clusters, biomolecules and chemical reactions [3].

Resonant two-photon ionization of atomic hydrogen has been examined thoroughly by theoreticians as a prime example which allows a detailed understanding of the underlying field-induced dynamics. Due to the resonantly driven bound-bound transition, the photoelectron spectra display Autler-Townes doublets and the total ionization probability exhibits a step-wise temporal evolution [1]. Other nonperturbative effects in strong laser fields were studied as well (see e.g. [4, 5] and references therein). Corresponding experimental studies are becoming feasible nowadays due to the advent of brilliant highfrequency photon sources such as advanced synchrotron beam lines and free-electron lasers.

Ionization may also occur through resonant photoexcitation of an autoionizing atomic level, which subsequently decays via Auger emission. In recent years this type of ionization mechanism, which relies on electronelectron correlations, has been investigated intensively, both theoretically [6 6 ] and experimentally, in systems consisting of two (or more) atoms, such as noble gas dimers [9, 10], metal oxides [11] or water molecules [12]. Here the radiationless deexcitation of one atom is accompanied with the ionization of another neighboring atom. This process is commonly referred to as interatomic Coulombic decay, ICD [6].

In this Letter, we study resonant two-photon ionization of a two-atom system which is exposed to an external electromagnetic field. In this situation, each atom is subject not only to the influence of the field but also to the interaction with the neighbor atom. Hence there are two pathways for ionization: either directly via resonant two-photon absorption at a single center, or mediated by ICD when each atom has been excited by singlephoton absorption (see figure 1). We shall show that the second mechanism can be remarkably efficient and may even dominate the direct channel by orders of magnitude. Moreover, the two-center channel exhibits very peculiar features with respect to its field dependence, temporal development and photoelectron spectra. In contrast to the two-center resonant photoionization considered in [8], the present process occurs for two identical atoms. It can, in principle, also exist in the case of two different atomic species provided that the latter ones possess a common dipole-allowed transition frequency.

Let us consider a system consisting of two atoms $(A$ and $B$ ) separated by a sufficiently large distance $R$ such that their individuality is basically preserved. Let each atom have an excited state reachable from its ground state by a dipole-allowed transition and the energy between these states be the same for both atoms. The atoms, which are initially in their ground states, are embedded in a resonant electromagnetic field. As seen from figure 1, already in the absence of the neighbor, each of the atoms can be ionized by absorbing two photons. However, due to ICD, photo ionization in the system of two atoms acquires interesting and qualitatively new features.

Assuming the atoms to be at rest, we take the position of the nucleus of atom $A$ as the origin and denote the coordinates of the nucleus of atom $B$, the electron of atom $A$ and that of atom $B$ by $\mathbf{R}, \mathbf{r}_{1}$ and $\mathbf{r}_{2}=\mathbf{R}+\boldsymbol{\xi}$, respectively, where $\boldsymbol{\xi}$ is the position of the electron of atom $B$ with respect to its nucleus.

The total Hamiltonian describing two atoms in the ex-

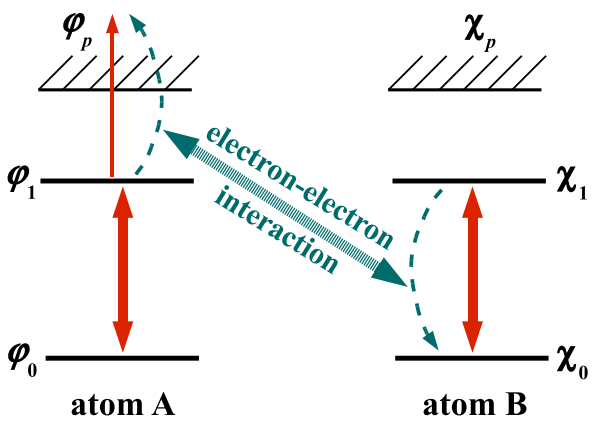

FIG. 1: Scheme of resonant two-photon single ionization of two atoms. In order not to overload the picture, ionization channels are shown for atom $A$ only. Ionization of atom $B$ proceeds analogously. 
ternal electromagnetic field reads

$$
H=\hat{H}_{0}+\hat{V}_{A B}+\hat{W},
$$

where $\hat{H}_{0}$ is the sum of the Hamiltonians for the noninteracting atoms $A$ and $B, \hat{V}_{A B}$ the interaction between the atoms and $\hat{W}=\hat{W}_{A}+\hat{W}_{B}$ the interaction of the atoms with the electromagnetic field.

For electrons undergoing electric dipole transitions the interatomic interaction $V_{A B}$ reads

$$
\hat{V}_{A B}=\frac{\alpha}{R^{3}}\left(e^{i k R}-i k R e^{-i k R}\right)-\frac{k^{2} \beta}{R} e^{i k R} .
$$

Here, $\alpha=r_{1 i} \xi_{j}\left(\delta_{i j}-3 R_{i} R_{j} / R^{2}\right), \beta=r_{1 i} \xi_{j}\left(\delta_{i j}-\right.$ $\left.R_{i} R_{j} / R^{2}\right), r_{1 i}$ and $\xi_{j}(i, j \in\{x, y, z\})$ are the coordinates of the electrons and a summation over the repeated indices is implied. Further, $k=\omega_{f i} / c$ where $\omega_{f i}$ is the transition frequency and $c$ the speed of light. Note that atomic units (a.u.) are used throughout unless otherwise stated.

The electromagnetic field will be treated as a classical, linearly polarized field, described by the vector potential $\mathbf{A}(\mathbf{r}, t)=c \mathbf{F}_{0} / \omega_{0} \cos \left(\omega_{0} t-\mathbf{k}_{0} \cdot \mathbf{r}\right)$, where $\omega_{0}=c k_{0}$ and $\mathbf{k}_{0}$ are the angular frequency and wave vector, and $\mathbf{F}_{0}$ is the field strength. The interaction $\hat{W}$ then reads

$$
\hat{W}=\sum_{j=1,2} \frac{1}{c} \mathbf{A}\left(\mathbf{r}_{j}, t\right) \cdot \hat{\mathbf{p}}_{j},
$$

where $\hat{\mathbf{p}}_{j}$ is the momentum operator for the $j$-th electron.

In what follows we shall assume that the electromagnetic field is not too strong such that field-induced transitions from bound states to the continuum are weak and, besides, one can neglect the influence of the field on the continuum. This also implies that only one of the centers, either $A$ or $B$, will be ionized with a non-negligible probability.

However, even a relatively weak, but resonant, electromagnetic field can very effectively couple bound states belonging to the same center. Therefore, for each center we first construct field-dressed bound states. Taking, as an example, the center $A$, assuming that the field is switched on suddenly at $t_{i}=0$, and using the rotatingwave approximation we obtain (see also [1, 2] )

$$
\begin{aligned}
\varphi_{+}(t) & =\frac{1}{z_{+}^{A}-z_{-}^{A}}\left(\left(z_{+}^{A}+\omega_{0}-\varepsilon_{1}\right) \exp \left(-i z_{+}^{A} t\right)\right. \\
& \left.-\left(z_{-}^{A}+\omega_{0}-\varepsilon_{1}\right) \exp \left(-i z_{-}^{A} t\right)\right) \varphi_{0} \\
& +\frac{W_{10}}{z_{+}^{A}-z_{-}^{A}}\left(\exp \left(-i z_{+}^{A} t\right)-\exp \left(-i z_{-}^{A} t\right)\right) \\
& \times \exp \left(-i \omega_{0} t\right) \varphi_{1}
\end{aligned}
$$

and

$$
\begin{aligned}
\varphi_{-}(t) & =\frac{W_{01}}{z_{+}^{A}-z_{-}^{A}}\left(\exp \left(-i z_{+}^{A} t\right)-\exp \left(-i z_{-}^{A} t\right)\right) \varphi_{0} \\
& +\frac{1}{z_{+}^{A}-z_{-}^{A}}\left(\left(z_{+}^{A}-\varepsilon_{0}\right) \exp \left(-i z_{+}^{A} t\right)\right. \\
& \left.-\left(z_{-}^{A}-\varepsilon_{0}\right) \exp \left(-i z_{-}^{A} t\right)\right) \exp \left(-i \omega_{0} t\right) \varphi_{1} .
\end{aligned}
$$

In these expressions $\varphi_{0}$ with an energy $\varepsilon_{0}$ and $\varphi_{1}$ with an energy $\varepsilon_{1}$ are the ground and excited states, respectively, of center $A$. Further,

$$
z_{ \pm}^{A}=\frac{1}{2}\left(\varepsilon_{0}+\varepsilon_{1}-\omega_{0} \pm \Omega_{R}\right),
$$

where $\Omega_{R}=\sqrt{\left(\varepsilon_{1}-\varepsilon_{0}-\omega_{0}\right)^{2}+4\left|W_{01}\right|^{2}}$ is the Rabi frequency and $W_{i j}=\left\langle\varphi_{i}\left|\mathbf{F}_{0} \cdot \hat{\mathbf{p}}_{1} /\left(2 \omega_{0}\right)\right| \varphi_{j}\right\rangle(i, j \in\{0,1\})$. The states (4) and (5) are orthogonal to each other and to the continuum states $\left\{\varphi_{\mathbf{p}}\right\}$ of center $A$, and are normalized to unity. At $t=0$ these field-dressed states reduce to $\varphi_{0}$ and $\varphi_{1}$, respectively. In the above description we have neglected the spontaneous radiative decay of the excited state $\varphi_{1}$ which in our case is justified as long as $\left|W_{01}\right| \gg \Gamma_{r}$, where $\Gamma_{r}$ is the radiative width of $\varphi_{1}$.

Expressions for the field-dressed bound states $\chi_{ \pm}(t)$ on the center $B$ with the corresponding quasi-energies $z_{ \pm}^{B}$ can be obtained from (4)-(何) by straightforward replacements.

Our consideration of the photoionization process will be based on the $S$-matrix formalism. Concentrating for the moment on the description of ionization of atom $A$ we write down the transition matrix element

$$
S_{f i}=-i \int_{t_{i}}^{t_{f}} d t\left\langle\psi_{f}^{A}(t)\left|\hat{V}_{A B}+\hat{W}_{A}\right| \Psi(t)\right\rangle .
$$

Here $\Psi$ is a solution of the Schrödinger equation for the total Hamiltonian $H$ and $\psi_{f}^{A}$ is a final channel corresponding to ionization of $A$. Note that neither $\hat{W}_{A}$ nor $\hat{V}_{A B}$ are included in the Schrödinger equation for $\psi_{f}^{A}$.

Taking into account that (i) the electromagnetic fieldinduced transitions to the continuum are weak, (ii) the field does not distort continuum states, (iii) the interatomic interaction at large distances is weak, and (iv) initially (at $\left.t_{i}=0\right)$ both atoms are in the ground states, one can approximate $\Psi(t)$ by $\varphi_{+}(t) \chi_{+}(t)$. Besides, the final state $\psi_{f}^{A}(t)$ can be represented by either one of the states $\varphi_{\mathbf{p}} \chi_{+}(t)$ and $\varphi_{\mathbf{p}} \chi_{-}(t)$. Here $\varphi_{\mathbf{p}}$ is the continuum state of center $A$ with an asymptotic momentum $\mathbf{p}$ and energy $\varepsilon_{p}=p^{2} / 2$.

As a result, ionization of center $A$ is described by the following transition amplitudes

$$
\begin{aligned}
& S_{\mathbf{p},+}(t)=-i \int_{0}^{t} d t^{\prime}\left\langle\varphi_{\mathbf{p}} \chi_{+}\left|\hat{V}_{A B}+\hat{W}_{A}\right| \varphi_{+} \chi_{+}\right\rangle \\
& S_{\mathbf{p},-}(t)=-i \int_{0}^{t} d t^{\prime}\left\langle\varphi_{\mathbf{p}} \chi_{-}\left|\hat{V}_{A B}\right| \varphi_{+} \chi_{+}\right\rangle .
\end{aligned}
$$

Since the final states $\varphi_{\mathbf{p}} \chi_{+}(t)$ and $\varphi_{\mathbf{p}} \chi_{-}(t)$ are orthogonal to each other, these amplitudes add up incoherently and for the probability of ionization of center $A$ we obtain

$$
P_{A}(t)=\int d^{3} \mathbf{p}\left(\left|S_{\mathbf{p},+}(t)\right|^{2}+\left|S_{\mathbf{p},-}(t)\right|^{2}\right) .
$$


Similar expressions hold for ionization of center $B$. Note that the time integrals in (8) are easily taken analytically. However, the resulting expressions are somewhat lengthy and will be given elsewhere.

Let us now turn to the discussion of some results following from the above expressions. Perhaps one of the most appropriate objects, where the process under consideration may occur, is a helium dimer exposed to a resonant field. However, for simplicity we restrict our attention here to the most fundamental two-atomic system consisting of two hydrogen atoms, which are initially in the ground state and are irradiated by an electromagnetic field with frequency resonant to the atomic $1 s-2 p$ transition. The internuclear distance $R$ is assumed to be such that the changes in the atomic levels, caused by the (full) interatomic interaction, remain smaller than even the natural width $\Gamma_{r}$ of the $2 p$ level in atomic hydrogen, which is fulfilled at $R \gtrsim 20$ a.u.. Since we also suppose that $\left|W_{01}\right| \gg \Gamma_{r}$ (which in case of hydrogen holds for $F_{0} \gtrsim 10^{-7}$ a.u.) these changes are negligible compared to those induced by the electromagnetic field. Therefore, one can indeed treat the ionization process by regarding the two-atomic system as consisting of two individual hydrogens whose atomic properties remain basically unchanged.

For not too strong electromagnetic fields and not too large separations between the atoms the two-center channel can strongly outperform the direct channel in photo ionization. Indeed, neglecting the retardation effects, one can show that for ionization of center $A$ the relative strength of the former with respect to the latter is determined by the ratio $\left(d_{B} /\left(R^{3} F_{0}\right)\right)^{2}$, where $d_{B}$ is the dipole moment of the bound-bound transition in center $B$. The case, when this ratio substantially exceeds unity, is illustrated in figure 2 where we present the probabil-
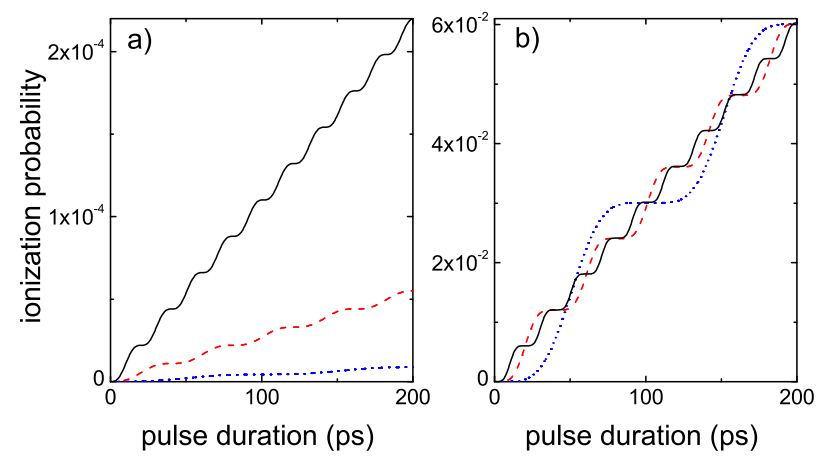

FIG. 2: Probability for single ionization of two hydrogen atoms separated by a distance $R=25$ a.u. given as a function of the electromagnetic pulse duration for zero detuning. The interatomic vector $\mathbf{R}$ is directed along the field polarization $\mathbf{F}_{0}$. Dot, dash and solid curves show the results for $F_{0}=2 \times 10^{-6}, 5 \times 10^{-6}$ and $10^{-5}$ a.u., respectively. a) the contribution of the direct channel only. b) the contribution of the two-center channel. ity $P_{A}(t)+P_{B}(t)$ for single ionization of two centers as a function of time. Since electron transitions into the continuum states occur only from the excited states the probability shows a non-monotonous behavior in which time intervals, when the ionization probability rapidly increases, are separated by intervals, when the probability remains practically constant, reflecting oscillations with the Rabi frequency of the electron populations between the ground and excited states in a resonant electromagnetic field.

Such a staircase behavior, related to the Rabi oscillations, is inherent for both the direct [1] and two-center channels of ionization (see figure 2a and $2 \mathrm{~b}$, respectively). However, since in the latter case for ionization to occur both centers have to be in the excited states, the "stairs" in the time development of the probability for ionization via ICD are more pronounced.

Note also that the probability for ionization via ICD may demonstrate a behavior which appears counterintuitive at first glance: the weakest field can lead to the highest instantaneous value of this probability (see figure 2 $\mathrm{b}$, pulse durations in the intervals around 75 and $175 \mathrm{ps})$. However, a simple analysis shows that such a behavior is in fact the consequence of (i) the dependence of the Rabi frequency on the field intensity and (ii) the field-independence of the probability for ionization via the two-center channel, temporally averaged over the inverse of the Rabi frequency.

The direct and two-center channels of photo ionization are in general characterized by different dependences on the field intensity. For instance, in the case of ionization by a monochromatic field, which is exactly resonant to the bound-bound transitions, the population probabilities for the excited bound states (averaged over

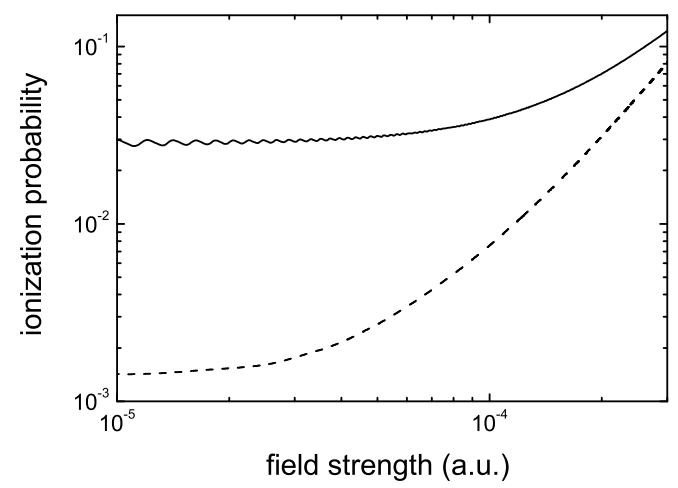

FIG. 3: Probability for single ionization of two hydrogen atoms separated by a distance $R=25$ a.u. given as a function of the electromagnetic field strength for a pulse duration of 100 ps. $\mathbf{R}$ is directed along $\mathbf{F}_{0}$. Solid curve: ionization by a monochromatic field with zero detuning. Dash curve: ionization by a nonmonochromatic field with bandwidth of $20 \mathrm{meV}$ and central frequency equal to the $1 s-2 p$ transition frequency in hydrogen. 
the inverse of the Rabi frequency) do not depend on the field intensity. As a result, the averaged probability for photo ionization via the direct channel, which involves absorption of an additional photon, is proportional to the field intensity. In contrast, the averaged probability for ionization due to the two-center mechanism is intensity-independent because it relies only on resonant bound-bound transitions and two-center electronelectron correlations. These two limiting dependences and a smooth transition between them are illustrated by the solid curve in figure 3. Similar results hold also for non-monochromatic fields (see dash curve in figure 3). At higher intensities, where the direct channel is more efficient, the ionization probability demonstrates a linear growth with intensity whereas at smaller intensities, where the two-center mechanism dominates, the ionization probability becomes almost a constant. The wiggles seen in figure 3 result from the Rabi-flopping dynamics.

In a resonant electromagnetic field each of the ground and excited levels of the centers $A$ and $B$ split into two sub-levels. Therefore, the direct ionization channel leads in general to two Autler-Townes lines (per atomic species) in the energy spectrum of the emitted electrons located at $\varepsilon_{p}=z_{ \pm}^{A}+2 \omega_{0}$ (see figure 㝳). Because of the splitting electron transitions between dressed bound states are characterized by three different transition energies. When these energies are transferred to the neighbor field-dressed center via ICD, one would obtain in general six energy lines (per atomic species) for the emitted electrons. For the emission from center $A$ they are given by $\varepsilon_{p}=z_{ \pm}^{A}+2 \omega_{0}, \varepsilon_{p}=z_{ \pm}^{A}+z_{-}^{B}-z_{+}^{B}+2 \omega_{0}$ and $\varepsilon_{p}=z_{ \pm}^{A}+z_{+}^{B}-z_{-}^{B}+2 \omega_{0}$. However, for identical centers only four of them have different energies (see figure $4 \mathrm{~b}$ ).

Since in the transition amplitude $S_{\mathbf{p},+}$ both direct and ICD ionization channels may lead to the same final states, they can interfere. As was mentioned, for identical atoms the direct and ICD channels result in two and four emis-

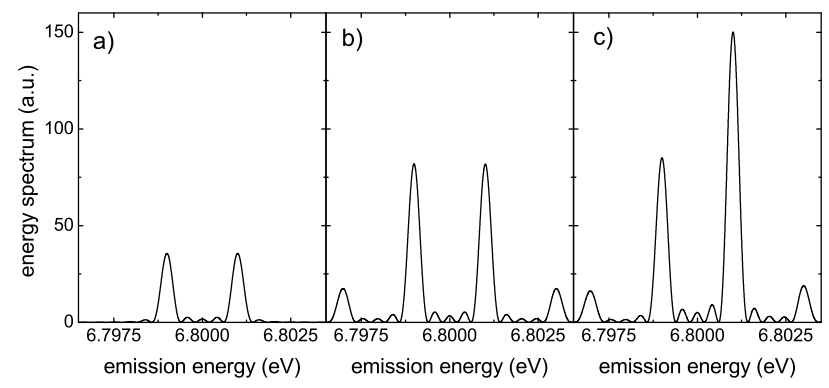

FIG. 4: Energy spectrum of electrons emitted in the process of single ionization of two hydrogen atoms separated by a distance $R=25$ a.u. at $F_{0}=10^{-4}$ a.u., zero detuning, pulse duration of 10 ps and $\mathbf{R}$ directed along $\mathbf{F}_{0}$. Panels a) and b) show the partial contributions of the direct and two-center mechanisms, respectively. Panel c) displays the total spectrum including the interference of the two channels. sion lines, respectively, in the energy spectrum of the emitted electrons. The former two coincide with the two central lines from the latter four. Therefore, for these two lines interference may occur. Indeed, comparing figures 4h, 4b and 4. we see that the total contribution of the two channels is not simply equal to the sum of their partial contributions. In particular, destructive and constructive interferences of these contributions in the amplitude $S_{\mathbf{p},+}$ occur, respectively, for the left and right central peaks.

In conclusion, resonant two-photon single ionization of a two-atom system was considered. For simplicity and in order to clearly reveal the basic underlying physics results for two hydrogen atoms were shown. It was found that, in a certain range of interatomic distances and external field strengths, the ionization is dominated by the two-center channel involving ICD. In this case, the mean ionization yield - averaged over one Rabi oscillation becomes independent of the applied field intensity. The instantaneous ionization probability shows a step-wise increase with time and a non-monotonous dependence on the field strength. The photo-electron spectrum comprises four lines. Its asymmetry reflects the interference between the direct and two-center ionization channels. An experimental observation of the predicted effects can be possible utilizing helium dimers, which were recently used successfully for studies of various aspects of ICD [10].

[1] P. L. Knight and P. W. Milonni, Phys. Rep. 66, 21 (1980)

[2] M.V. Fedorov and A.E. Kazakov, Prog. Quant. Electr. 13, 1 (1989).

[3] J. C. Miller et al., Phys. Rev. Lett. 45, 114 (1980); G. von Helden et al., Phys. Rev. Lett. 79, 5234 (1997); E. Nir et al., Chem. Phys. Lett. 355, 59 (2002).

[4] K. Rzazewski and J. H. Eberly, Phys. Rev. Lett. 47, 408 (1981); G. S. Agarwal et al., ibid. 48, 1164 (1982).

[5] M.G. Girju et al., J.Phys. B 40, 4165 (2007)

[6] L. S. Cederbaum et al., Phys. Rev. Lett. 79, 4778 (1997).

[7] V. Averbukh, I. B. Müller, and L. S. Cederbaum, Phys. Rev. Lett. 93, 263002 (2004); K. Gokhberg et al., Europhys. Lett. 72, 228 (2005)

[8] B. Najjari, A. B. Voitkiv and C. Müller, Phys. Rev. Lett. 105, 153002 (2010)

[9] T. Jahnke et al., Phys. Rev. Lett. 93, 083002 (2004); Y. Morishita et al., ibid. 96, 243402 (2006).

[10] N. Sisourat et al., Nature Phys. 6, 508 (2010); T. Havermeier et al., Phys. Rev. Lett. 104, 133401 (2010).

[11] A. Kay et al., Science 281, 679 (1998); F.J. Garcia de Abajo et al., Phys. Rev. Lett. 82, 4126 (1999)

[12] T. Jahnke et al., Nature Phys. 6, 139 (2010); M. Mucke et al., ibid. 6, 143 (2010). 